\title{
213 PHYSICAL INACTIVITY AS A GLOBAL RISK FACTOR FOR CHRONIC DISEASES IN WOMEN
}

Poonam Lata Research Scholar, MD University, Rohtak, Haryana, India

10.1136/bjsm.2010.078725.213

Social inequality, poverty and inequitable access to resources, including healthcare, result in a high burden of non-communicable diseases among women worldwide. Regular physical activity can improve women's health and help prevent many of the diseases and conditions that are major causes of death and disability for women around the world. Many women suffer from disease processes that are associated with inadequate participation in physical activity.

Facts

- Cardiovascular diseases account for one third of the death in women worldwide.

- Diabetes affects more than 70 million women in the world and its prevalence is projected to double by 2025 .

- Osteoporosis is most prevalent in post-menopausal women.

- Physical inactivity is more common among women than men.

Benefits of physical activity

- Reduces the risk of dying from coronary heart disease and of developing high blood pressure, colon cancer and diabetes.

- Helps maintain healthy bones, muscles and joints.

- Helps control weight, build lean muscle and reduce body fat.

- Helps control joint swelling and pain associated with arthritis.

- May enhance the effect of oestrogen replacement therapy in decreasing bone loss after menopause.

- Reduces symptoms of anxiety and depression and fosters improvement in mood and feelings of well-being.

- Reduces the risk of breast cancer in women.

Reasons for physical inactivity in women

- The income of women is often lower than that of men and therefore the costs of access to physical activity facilities may be a barrier.

- Agreement may be required from senior members of the household who control household resources before a woman can engage in physical activity.

- Women often have a workload in the home and care-giving roles for other family members, which may limit the time available for them to engage in physical activity.

- Women who have limited mobility may be unable to travel to health centres or physical activity facilities.

- Cultural expectations may restrict the participation of women in certain forms of physical activity.

- Lack of motivation and lack of awareness of physical education. 